\title{
L'ANTHROPOLOGIE PHILOSOPHIQUE : UN HÉRITAGE DES MÉTAPHYSIQUES HUMANISTES POUR LES SCIENCES HUMAINES *
}

La " Nature ", la "Liberté " et l' " Esprit " sont les trois grands paradigmes métaphysiques sous lesquels les philosophies du xvme et du XIXe siècle tentèrent de penser l'histoire de l'humanité civilisée comme une réalisation de la raison dans le monde. Il s'agit de trois complexes conceptuels qui devaient exprimer chacun une sorte de code logique stylisant un mode idéal sur lequel s'accomplirait, en tant qu' " histoire universelle ", la synthèse du réel et du rationnel.

Aujourd'hui, nous avons certes largement abandonné de telles représentations, et la " Nature », la "Liberté ", l' « Esprit ", sont tout au plus les index sous lesquels nous pouvons repérer des légalitès différentes, autonomes, pour les différentes dimensions d'une modernisation wébériennement conçue comme rationalisation de la réalité sociale : l'économie, la politique, la culture sont les dimensions évolutives de la réalité sociale au sens large, dont chacune conserve en sa logique quelque chose de la raison qu'intentionnaient les trois paradigmes des' philosophies de l'histoire. Par leur contenu rationnel respectif comme par la délimitation réciproque des domaines d'objets qui leur correspondent, ces trois paradigmes idéalistes représentent un potentiel conceptuel qui a pu, sur la voie des sciences humaines, être exploité au niveau intermédiaire d'une anthropologie philosophique. Leur conceptualité peut aujourd'hui être mobilisée dans des contextes scientifiques, et indépendamment des contextes proprement philosophiques dans lesquels ils furent élaborés.

J'aimerais ici examiner successivement ces trois paradigmes métaphysiques du point de vue de leurs implications anthropologiques. Je partirai de l'exemplification qui en fut donnée notamment dans l'idéa-

\footnotetext{
* Ce travail prend place dans le cadre d'une recherche soutenue par la Fondation Alexander von Humboldt. Les hypothèses esquissées dans cet article seront développées dans un prochain livre à paraitre chez A. Fayard.
}

Revue de synthèse : IVe S. No 4, octobre-décembre 1986. 
lisme allemand, chez Kant, Fichte et Hegel (I). De là, j'analyserai certaines présuppositions importantes du paradigme de l'Esprit, tel qu'il avait été initialement travaillé par le jeune Hegel dans le cadre d'une anthropologie philosophique : c'est là que la culture d'une époque doit se réfléchir comme le résultat d'activités déterminées qui engagent chacune à sa manière un aspect de l'universel, ou encore, une forme de la rationalité. Ces différents modes d'activités organisées dans les « milieux » du langage, du travail, de l'interaction, supposent des rapports au monde différenciés, et ils ont fait l'objet de systématiques susceptibles de faire retour dans la discussion contemporaine sur les fondements d'une théorie de la société (II).

Kant ${ }^{1}$ avait défini deux domaines de "législation " (ou d'objectivité) rigoureusement séparés l'un de l'autre : tandis que la "législation de la nature " détermine ce que l'entendement peut objectiver du point de vue de l'intelligence connaissante (ce qui est), la "législation de la liberté " représente ce que la raison peut exiger d'inconditionné pour la volonté agissante (ce qui doit être). Il s'ensuivait, chez Kant, que seule la Nature fournit la légalité pour ce qui se produit dans la réalité phénoménale ou empirique, tandis qu'en principe la Liberté ne légifère qu'au niveau de l'Idée, dans le champ nouménal. C'est pourquoi Kant s'est appuyé sur le seul concept de "nature " dans sa tentative de penser l'histoire comme un procès continu de rationalisation de la réalité sociale. Toutefois, il voulait penser l'histoire humaine sans perdre de vue la fin qu'exige la raison pratique, à savoir la réalisation de la Liberté au niveau des institutions politiques et des normes juridiques réglant les relations entre les individus ainsi qu'entre les Etats

1. Immanuel KaNT, Idée d'une histoire universelle au point de vue cosmopolitique, suivi de : Réponse à la question : qu'est-ce que les Lumières?, suivi de : Le Conflit des facultés, in Kant. La philosophie de l'histoire (recueil de textes choisis), Paris, Denoël-Gonthier, 1947. Également, ID., Théorie et pratique, Paris, Vrin, 1972; ID., Vers la paix perpétuelle. Essai philosophique, Paris, Vrin, 20 éd. 1975. Pour des interprétations concurrentes de la philosophie politique de Kant dans ses écrits sur l'histoire universelle, Alexis PHILoNENKO, Théorie et praxis dans la pensée morale et politique de Kant et Fichte en 1793, Paris, Vrin, 1976 (Première partie) ; ID., « L'idée de progrès chez Kant », Revue de métaphysique et de morale, 4, 1974 ; Luc FERRY, Philosophie politique. T. II : Le Système des philosophies de l'histoire, Paris, P.U.F., 1984, chap. III ; ID., « Kant. Critique de la faculté de juger ", in François Chatelet, Olivier Duhamel, Evelyne Pisier, eds, Dictionnaire des auvres politiques, Paris, P.U.F., 1986, p. 401 sqq. Egalement, une piste critique intéressante, Jürgen Habermas, "Kant. La Publicité médiatrice de la politique et de la morale ", in J. HABERMAS, Strukturwandel der Oeffentlichkeit. Untersuchungen zu einer Kategorie der bürgerlichen Gesellschaft, Neuwied-Berlin, Luchterhand, 1962, trad. fr. par Marc B. DE LAUNAY, L'Espace public, Paris, Payot, 1978, chap. IV, sous-section 13, p. 112 sqq. 
eux-mêmes. D'un autre côté, les moyens de cette fin ne peuvent, dans leur enchaînement, se soustraire aux " lois universelles de la nature ", de sorte que si le but est bien la Liberté, c'est la Nature qui devra réaliser, suivant ses voies propres, les desseins que par ailleurs formerait une volonté libre. C'est à la Nature que revient notamment la tâche de réaliser la " République universelle ", l' "État cosmopolitique " régi par les lois de la liberté.

Il importe de se demander comment le " règne du Droit " pourra se réaliser sans que l'on doive faire appel à une autre instance qu'à la dynamique naturelle des penchants égoïstes des hommes. De là, on pourrait espérer que cette légalité, produite par le jeu des passions naturelles, le " jeu des penchants " immédiats, se convertisse " en un tout moral ", grâce aux progrès de la culture et des Lumières. Kant ne dit pas précisément comment la nature se raffinerait progressivement dans la culture pour faire accéder l'homme à la moralité. Mais il suppose, comme Hobbes, une situation de départ pré-politique, caractérisée par la guerre de tous contre tous, de sorte que les hommes, plongés dans une détresse extrême, soient acculés, contre leur désir d'indiscipline, à se soumettre à un " ordre légal ". Sous la garantie que seul peut offrir au pacte d'association un pacte de soumission, l' « enclosure " juridique d'une "association civile" permet de juguler les débordements autodestructeurs d'interactions entre des natures agonistiques. Or, cette logique interactionnelle du conflit et de la guerre, pourtant si néfaste lorsqu'elle est débridée en dehors d'un ordre légal, produit, au contraire, les meilleurs effets, une fois enclose dans cet ordre, aussi despotique soit-il au départ : la logique agonistique, cette "donnée naturelle ", se sublime dans la compétition réglée, la concurrence entre les individus pour les enjeux sociaux de pouvoir et de richesse, si bien que, sans le vouloir expressément, chaque individu œuvre pour le progrès matériel et culturel de l'ensemble. Kant utilise à cet égard la métaphore suggestive des arbres qui ne poussent beaux et droits que dans la forêt, car c'est là qu'ils essaient de se dépasser pour atteindre l'air et la lumière. C'est ainsi que, sous la condition d'une discipline sociale minimum, la "nature " peut d'elle-même "multiplier les produits de la légalité ". Le droit résulterait alors des ajustements cumulatifs d'une interaction stratégique régulée par la donnée encore naturelle de l'intérêt bien compris, mais non point d'exigences morales, communicationnellement partagées, politiquement spécifiées, et conceptuellement informées par une claire intelligence du bien en général. Il résulterait, autrement dit, d'une logique de la " nature " et non d'une logique de la "liberté ".

Certes, une voie autoritaire est possible pour imposer la liberté, 
ainsi qu'avait pu le révéler l'expérience de la Révolution française. Et cette voie volontariste qui caractérisa le processus révolutionnaire français relève bien, pour sa compréhension, des catégories de la liberté, si l'on entend par là une création juridique dont l'idée morale intransigeante fut appliquée à la réalité jusqu'aux extrêmes conséquences. Là, le processus est sans doute tellement marqué par la problématique du passage révolutionnaire de la "théorie " (les principes du Droit naturel) à la " pratique " (la réalisation politique de ces principes dans le droit positif) qu'il semble inopérant de vouloir en rendre compte dans les termes d'un mécanisme aveugle, infra-politique, c'està-dire selon les catégories de la nature. En fait, si Kant faisait fond sur l'immanence d'une contrainte naturelle, c'était aussi pour ne pas avoir à justifier la transcendance d'une violence politique : non seulement le despotisme d'un État qui imposerait le Droit rationnel par la violence révolutionnaire contredit tout autant l'idée que l'on se fait de la liberté, mais, en outre, le recours à la force pacifiante d'un ordre légal instauré par la violence politique ferait dès le départ éclater le cadre épistémologique d'une histoire conçue sur le strict modèle d'une dialectique de la Nature. Le problème refait surface, lorsque la « dialectique de la nature " révèle sa limite dans l'impuissance à expliquer comment pourrait se maintenir le respect du droit, une fois que, d'une façon ou d'une autre, ce dernier a pu être instauré positivement : il faut donc l'autorité d'un chef. C'est à une aporie que se heurte finalement Kant : ni la confiance libérale dans les lois de la nature, ni l'intransigeance jacobine sur les lois de la liberté ne sauraient pleinement satisfaire la raison. Kant doit alors faire appel aux vertus pédagogiques non violentes de la Publicité (Oeffentlichkeit) et au progrès des Lumières. La Publicité, "usage public de la raison ", apparaît comme la méthode même de l'Aufklärung, tandis que cette dernière s'intègre subrepticement à la logique de l'histoire. En effet, requérir les vertus formatrices d'une Oeffentlichkeit et d'une Aufklärung censées former l'opinion à la raison dans un processus unissant la volonté et l'intelligence, c'était bien faire fond, pour l'histoire, sur une autre logique que celle de la nature : c'était faire signe vers une logique de l'esprit.

Kant ne pouvait pas développer cette logique comme telle, car il se maintenait à un concept restreint de la réalité phénoménale en voulant la penser sous l'idée de Nature, ainsi que l'y oblige le système de sa propre philosophie. Kant ne pouvait entreprendre une phénoménologie de la Liberté, car ce qui est phénoménal n'appartient pour lui qu'aux lois de la Nature. Et ce n'est donc que par une sorte de " ruse de la Nature " que la Raison peut se réaliser dans l'histoire. Cette position ne s'explique pas seulement par une anthropologie pessimiste 
qui préférait partir de l'égoïsme des hommes. D'ailleurs, Kant admettait aussi que la nature humaine fût quelque part accessible à la moralité, et, en ce sens, "digne d'amour". En fait, si Kant ne tient pas compte de la façon dont les hommes pourraient agir selon les pures déterminations morales d'un être libre, c'est surtout pour deux raisons distinctes. La première raison tient à la nature même du politique, et elle fut souvent négligée par les interprètes de Kant : même si chaque individu pris individuellement était convaincu par la nécessité de soumettre la communauté sociale à une constitution politique conforme aux principes d'un droit rationnel, cette "unité distributive" de la volonté de tous ne suffirait pas, car il faut, en matière politique, la force qui rassemble les volontés éparses, l' « unité collective" de la volonté de tous. C'est à vrai dire ce problème, en apparence bien modeste, qui fait surgir l'obstacle épistémologique le plus puissant à l'efficace politique de la liberté intérieure, mais aussi, du mécanisme naturel. Cependant, il est une raison plus massive, qui tient quant à elle aux admissions fondamentales de l'ontologie kantienne ; même si, d'un point de vue métaphysique, nous sommes autorisés à postuler la liberté humaine, les manifestations sensibles de cette liberté, que sont les actions humaines, sont pour Kant des phénomènes qui, comme tels, seraient à considérer exactement au même titre que n'importe quel autre phénomène de la nature. Kant en voulait pour signe le fait qu'au niveau macro-historique les comportements sociaux obéissent à des constantes statistiquement pertinentes, dont la logique d'ensemble échappe au libre arbitre de chaque individu pris séparément.

Or, Fichte ${ }^{2}$ a précisément contesté que l'on puisse regarder les actions humaines comme des phénomènes de la Nature. Si les actions humaines peuvent être immédiatement perçues et comprises comme des manifestations sensibles de la Liberté, cela signifie aussi que la Liberté, loin d'être reléguée au royaume des noumènes, se phénoménalise dans des formes de vie qui relèveraient précisément d'une herméneutique de l'humain. Le domaine des actions humaines, qui pour Kant constituait déjà la trame de l'histoire, doit alors, en fonction de

2. Johann Gottlieb FichTE, Fondement du droit naturel selon les principes de la doctrine de la science (1796-1797), prés., trad. et notes par Alain Renaut, Paris, P.U.F., 1984. Pour des interprétations de la pensée du jeune Fichte, cf. A. PHILONEnko, op. cit. supra n. 1 ; ID., La Liberté humaine dans la philosophie de Fichte, Paris, Vrin, rééd. 1980 ; également, dans cette ligne, A. Renaut, Le Système du droit. Philosophie et droit dans la pensée de Fichte, Paris, P.U.F., 1986 ; encore, L. FERRY, op. cit. supra n. 1, section 2, en particulier, chap. III ; spécifiquement, sur le lien entre droit et communication dans le premier système de Fichte, R. LAUTH, "Le problème de l'interprétation chez J. G. Fichte ", Archives de philosophie, juil.-déc. 1962. 
Fichte, être abordé par la connaissance comme un donné empirique spécifique qu'il nous est impossible de saisir sur le modèle d'un pur mécanisme (éventuellement finalisé par une téléologie quasi naturelle). Car le sens qui s'y exprime manifeste l'autonomie d'un être capable d'agir suivant des représentations. Or, c'est là le caractère d'un être susceptible d'une histoire en général. Fichte laisse ainsi apercevoir une autre possibilité épistémologique de comprendre l'histoire que celle que Kant avait lui-même indiquée : dans la mesure où l'histoire n'est plus soumise à une logique de la Nature, mais se fait plutôt connaître comme manifestation sensible et comme expression signifiante de la Liberté, elle ne relève plus au premier titre d'une connaissance nomologique, mais d'une connaissance herméneutique. Fichte n'en a pas tiré les conclusions explicites au niveau d'un sujet transcendantal autre que le sujet transcendantal kantien, lequel ne fait apparaître qu'une compétence d'objectivation présupposant la seule relation sujet/objet - mais ni la relation intersubjective sujet/sujet, ni la relation autoréflexive du moi à lui-même. Fichte implique en revanche le caractère transcendantal d'une autre relation cognitive : la relation sujet/sujet, qui seule fonde l'accès au sens des expressions de la subjectivité. La justification anthropologique de cet autre donné phénoménal est obtenue chez Fichte au moyen d'une phénoménologie de l'ontique naturelle caractérisant les différents stades de l'être, depuis la matière inorganique jusqu'à la "vie ", en passant par l'organisation. Dans cette hiérarchie des étants naturels, la vie se désigne comme individualité, c'est-à-dire comme une forme d'existence capable d'une activité autonome. La plante, par exemple, n'appartient pas à proprement parler à la "vie ", car il lui manque l'autonomie du mouvement et de l'action : un arbre peut être greffé, et s'accroître ainsi de nouveaux éléments. Mais il n'est pas articulé. L'articulation manifeste l'autonomie de l'être vivant dont la liberté se signale à ce niveau par une aptitude à la manipulation des choses.

Fichte consacre en outre les plus belles phrases à ce qui se lit sur un visage humain. Son anthropologie permet alors de caractériser la liberté à deux niveaux logiques distincts: tandis que l'oeil manifeste éventuellement par le regard la liberté d'un être naturel comme étant animé par la représentation, la main manifeste aussi la liberté, mais à un autre niveau de compétence et d'activité : le niveau de l'instrumentation. En d'autres termes, les lieux où la liberté humaine se manifeste de façon sensible perceptible sont d'une part l'expression et d'autre part l'action. Au niveau de l'expression, la liberté est langage; son histoire pourrait alors être reconstruite dans les termes d'une genèse logique de la représentation. Au niveau de l'action, la liberté est techni- 
que; son histoire pourrait alors être reconstruite dans les termes d'une logique de l'instrumentation. Mais ce modèle d'action permet-il de connaître la façon dont la liberté doit être acquise au niveau politique?

Ce niveau est, en effet, celui d'une institutionnalisation de la Liberté dans le Droit. Et à ce titre, l'histoire de la liberté humaine incarnée socialement devrait plutôt procéder d'une logique de l'interaction. C'est d'ailleurs cette logique que Kant avait indiquée, bien qu'il la subsumât sous l'idée d'une Nature dont le « plan " rejoindrait les desseins de la Liberté. Or, Fichte opère plutôt une démarche inverse : ce n'est pas la Nature qui prépare la Liberté ; c'est la Liberté qui pose la Nature. Cependant, c'est dans une logique de l'instrumentation que fondamentalement, pour Fichte, la Nature peut être posée comme objet de la Liberté : la Nature, ni bonne ni mauvaise, est traitée par la Liberté comme un neutre - et cela peut même s'entendre au sens grammatical d'un "il " (es). Dans cette perspective logique, le Moi se pose comme Liberté en s'opposant à lui-même comme Nature au sens d'une matière neutre sur laquelle un travail est toujours possible. Le Moi est essentiellement Liberté, mais il ne s'éprouve et ne se connaît comme tel, c'est-à-dire comme activité déterminante, qu'en s'opposant à lui-même sa propre nature comme matière neutre sur laquelle il peut agir. Ainsi se fait-il exister comme Liberté. La Liberté s'opère ici dans une relation à la nature, où cette dernière est traitée comme un pur objet, un neutre au sens grammatical du terme. Cependant, la nature est représentée sous son double aspect : à la fois nature externe (hors de nous) et nature interne (en nous). Le travail de la liberté sur la nature interne est l'activité morale. C'est pourquoi ce n'est pas seulement dans l'acte instrumental, la technique, que la Liberté s'affirme dans une relation je/il : bien que transposée sur le mode relationnel « je/je», une telle relation se trouve aussi présupposée dans l'acte moral, l'éthique.

Or, ceci pose le problème d'une formulation de la Liberté dans le Droit. On voit, en effet, que le Droit ne peut plus être d'essence morale, car la relation je/il, même modalisée dans un " je/je", ne fonde pas l'intersubjectivité. À cet égard, la parole de Fichte : « Pas de Toi, pas de Moi, pas de Moi, pas de Toi " ne pose pas nécessairement le principe de l'intersubjectivité. Elle pourrait encore renvoyer à un processus solipsiste de reconnaissance a parte d'un Alter ego. Fichte évoque cependant l'image sublime de deux regards qui se plongent l'un dans l'autre. Ce n'est plus le choc sur un objet, mais la rencontre entre deux sujets : le « je " reconnaît dans le "tu " cet autre « je " capable de le reconnaitre lui-même dans cette capacité... 
de sorte que le Moi dépasse la réflexivité du je-subjectivité dans la spéculativité du je/tu-intersubjectivité (laquelle est au demeurant, pour Fichte, la condition même de la conscience de soi).

Mais s'il doit être le départ du Droit, l' « appel » à limiter ma propre liberté pour faire place à celle que me signifie l'expression d'un autre qui me reconnaît présuppose que l'Alter comme l'Ego puisșent s'accorder pour valoriser ce signe naturel de la liberté humaine comme la norme fondamentale d'une communauté politique. Or, l'interaction est ici immédiate, directe, anhistorique. Elle n'est pas médiatisée par l'élément commun d'une culture fixant le symbole dans lequel les deux protagonistes peuvent prendre conscience d'eux-mêmes comme d'un " nous " déterminé. Ce qui fait défaut chez Fichte, à savoir le medium objectif de l'intersubjectivité, renvoie à tout un processus de formation, sans lequel la conscience de soi d'un nous n'a pas d'incarnation. C'est précisément à ce niveau que se justifie le paradigme de l'Esprit.

C'est $\mathrm{Hegel}^{3}$ qui a mis explicitement en place ce troisième paradigme pour penser l'histoire. "Plan de la Nature », chez Kant, " performance de la Liberté ", chez Fichte, l'histoire est essentiellement, chez Hegel, « mouvement de l'Esprit ». Ce paradigme fut développé par Hegel dans deux contextes différents : d'abord dans sa Realphilosophie d'Iéna, puis dans sa Phénoménologie de l'esprit. Il s'agit, dans le premier cas, d'expliciter les expériences fondamentales où s'élaborent les déterminants d'une communauté morale et politique : c'est la représentation dans le milieu du langage, le travail dans le milieu de l'outil, l'interaction dans le milieu de la famille, puis de la société civile. Cette conceptualité ressort des deux « philosophies de l'esprit " de la période d'Iéna, et elles sont elles-mêmes préfigurées par le Système de la vie éthique. Cependant, les trois logiques (représentation, travail, interaction) précédemment reconstruites en deux procès ( " théorique » et " pratique ») de formation de la conscience - ces trois logiques de l'expérience thématisées dans les leçons d'Iéna se trouvent

3. Georg Wilhelm Friedrich Hegel, La Phénoménologie de l'esprit, trad. et notes de Jean HyPPOLITE, 2 vols, Paris, Aubier Montaigne, 1939 et 1941 ; ID., La Raison dans l'histoire. Introduction à la philosophie de l'histoire, trad. nouv., introd. et notes par Kostas Papaloannou, Paris, Plon, 1965, chap. II, « La réalisation de l'esprit dans l'histoire". Pour la période d'Iéna (1801 à 1806), cf. les traductions et commentaires de Jacques TAMiNiauX, in G.W.F. Hegel, Système de la vie éthique, Paris, Payot, 1976, et J. Taminiaux, Naissance de la philosophie hégélienne de l'État. Commentaire et traduction de la Realphilosophié d'léna (1805-1806). Pour la période 1803-1804, G.W.F. HegEL, Jenaer Systementwiurfe. I : "Philosophie des Geistes", Leipzig, Felix Meiner, 1975 ; cf. éventuellement, la traduction et la présentation de Guy Planty-Bonjour, in G.W. F. Hegel, $L a$ Première philosophie de l'esprit, Paris, P.U.F., 1969. 
simplement présupposées dans la Phénoménologie de l'esprit, et comme dépassées sur le mode d'une autoréflexion qui se met elle-même en scène comme auto-mouvement du Concept se réalisant à travers les étapes succéssives d'un développement nécessaire où se lit le code de l'histoire universelle. À la différence de la Phènoménologie, la « philosophie de l'esprit " d'Iéna n'articulait pas l'expérience prototypique de la conscience qui se forme à une logique de l'histoire universelle dans laquelle se succéderaient les différents moments ou différentes « déterminités " du Concept. Toutefois, ce qui reste commun à la philosophie hégélienne, c'est le principe essentiel suivant lequel la forme de vie dans laquelle s'exprime l'humanité est l'Esprit. Pourquoi la réalité humaine, et partant, l'histoire, devraient-elles alors être pensées sous le paradigme de l'Esprit?

L' " Esprit " peut s'entendre très simplement au sens où l'on parle, par exemple, de l'esprit d'un peuple, d'une époque, ou même d'une équipe. Ce que Hegel nommait "esprit objectif" est l'ensemble constitué par les normes, les valeurs, les symboles, les institutions qui marquent la spécificité culturelle d'une communauté d'individus. Cette structure commune traduit une identité, une individualité qui représenterait le contexte d'intercompréhension à l'intérieur duquel les individus peuvent s'entendre entre eux et coordonner leurs actions de façon réciproque. L'Esprit peut être alors défini comme

« la communication des individus singuliers dans le milieu d'un universel qui fonctionne comme la grammaire d'une langue par rapport aux individus qui la parlent ou comme un système de normes en vigueur par rapport aux individus qui agissent $" 4$.

On pourrait dire qu'il est un " transcendantal " à sa manière, mais un transcendantal historique, et qui vaut de plus comme un transcendantal de l'intersubjectivité. C'est dans ce milieu que les sujets peuvent prendre conscience de ce qui les relie les uns aux autres et chacun à la communauté qu'ils forment, c'est-à-dire se poser comme des personnes individuées, au regard de ce qui à la fois les unit et les médiatise. Le concept hégélien d' " esprit objectif » fut repris par l'historisme et le néo-kantisme allemands, et il sert plus généralement de paradigme fondamental aux sciences historico-herméneutiques d'ailleurs appelées aussi "sciences de l'esprit" (Geisteswissenschaften). Selon ce paradigme, les différentes cultures et les différentes époques.

4. J. Habermas, "Travail et interaction. Remarques sur la "philosophie de l'esprit" de Hegel à Iéna ", in ID., La Technique et la science comme idéologie, préf. et trad. de l'allemand par Jean-René Ladmiral, Paris, Gallimard, 1973, p. 170-171. 
peuvent être comprises et interprétées comme un "texte" où s'exprime le génie propre d'une individualité collective. L'Esprit est donc le concept objectif que se donne à elle-même une forme de vie historique. Ce concept est concret au sens où il est le langage propre dans lequel cette forme de vie se communique à soi et aux autres. Il est concret également au sens où ce langage est l'élaboration et la mise en forme symboliques des résultats de l'activité et de l'expérience sociales. Il est la structure qui réfléchit son propre processus. Or, c'est justement parce que les sociétés historiques sont capables de se représenter elles-mêmes dans un système symbolique qui résume la totalité des expériences accédant à l'existence sociale que l'évolution de ces sociétés peut être saisie comme vie de l'esprit. Précisément, parce que ce système symbolique de la culture au sens large est le langage naturel dans lequel une société se dit elle-même, et se donne à comprendre pour elle et pour autrui. L'Esprit est le paradigme qui, plus que celui de la Nature et plus que celui de la Liberté, conviendrait pour penser l'histoire, parce qu'il est la mise en forme culturelle, c'est-à-dire le texte symbolique expressif sans lequel l'existence d'une société ne pourrait même pas se communiquer à ses membres ainsi qu'aux ressortissants d'autres cultures. Sans l'Esprit, les sociétés humaines seraient pour ainsi dire privées du langage du "nous" - et le "nous", une simple existence sans concept, une intuition aveugle.

Que fit Hegel de cette possibilité philosophique qu'il avait lui-même ouverte? Quel traitement la problématique de la constitution d'une conscience de soi pour le nous reçut-elle de sa philosophie de l'esprit? Dans la Realphilosophie telle que l'avaient esquissée les leçons d'Iéna, l'esprit peut apparaitre comme une " œuvre " dont l'auteur ne serait cependant aucun sujet particulier, parce qu'en principe cette " œuvre " serait la constitution même de l'intersubjectivité. C'est du moins ainsi, qu'après Hegel, le fit comprendre le néo-hégélien Theodor Litt ${ }^{5}$; et c'est ainsi que l'on pourrait également reconstruire le concept de l'esprit objectif à partir de la phénoménologie interactionniste héritière de Edmund Husserl dans la sociologie et dans la psychologie sociale (Georg Herbert Mead et Alfred Schütz) ${ }^{6}$. Mais chez Hegel lui-même, après l'abandon de la systématique d'Iéna au profit

5. Theodor LITT, Individuum und Gemeinschaft, Leipzig, 1926 ; ID., Mensch und Welt : Grundlinien einer Philosophie des Geistes, München, 1971.

6. Georg Herbert MEAD, Mind, Self and Society, Chicago, Ch. W. Morris, 1934; ID., Philosophie der Sozialität, Frankfurt am Main, H. Kellner, 1969 ; Alfred SchUTZ, Der sinnhafte Aufbau der sozialen Welt, Wien, 1932 ; ID., "Das Problem der transzendentalen Intersubjektivität bei Husserl ", Phil. Rundschau, 5, 1957, p. 81 sqq.; ID., Theorie der Lebensformen, Frankfurt am Main, 1981 ; également, A. SCHÚtz, Th. LuckmanN, Strukturen der Lebenswelt, Frankfurt am Main, 1979. 
du Système, le rapport entre le concept d'esprit objectif et ce qui est visé à travers lui, à savoir la conscience de soi du nous comme tel, ce rapport ne fut pas approfondi dans le sens d'une théorie de la communication (Jürgen Habermas) ${ }^{7}$. À lire les textes, même tardifs, de Hegel sur l'histoire, il s'avère que celui-ci n'abandonna jamais l'idée d'une histoire humaine conçue comme histoire de la liberté. Cependant, Hegel jugea finalement que le niveau auquel le mouvement de l'histoire pouvait être connu par la raison comme le mouvement d'une Liberté qui s'explicite dans l'Esprit ne pouvait être le niveau subjectif auquel les individus agissent dans l'histoire pour des fins le plus souvent particulières, mais seulement le niveau objectif de l'Esprit qui se réfléchit en lui-même dans un mouvement autonome, et surmonte ainsi l'aliénation qu'il doit produire en s'objectivant lui-même, afin d'exister historiquement comme être-là dans l'apparence objective. Considéré suivant cette dialectique de l'aliénation/réconciliation, l'Esprit reprend la totalité des objectivations de sa propre activité, rend celles-ci conscientes et les perce à jour, et, dans cette autoréflexion, laisse derrière lui comme un corps mort la forme de vie objectale dont les limites péremptoires définissent à chaque fois et pour chaque époque le contexte objectif normatif à l'intérieur duquel se meuvent les activités humaines.

La limitation qui en résulte pour la Liberté des individus dans l'histoire n'est alors dépassée qu'au niveau d'une autoréflexion dans laquelle l'Esprit absolu se connaît lui-même et supprime son extériorité à soi. Mais les consciences naturelles particulières des individus socialisés ne participent qu' "objectivement " de cette conscience de soi. En effet, la conscience de soi du "nous " n'est pas ici obtenue dans l'intersubjectivité réflexive d'une communication où les sujets de format ordinaire pourraient s'entendre pour une définition coopérative de ce qui leur est commun, situer ainsi leur pratique sous l'idée d'une histoire raisonnable dont ils seraient les véritables auteurs grâce au rappel d'une "raison" qui, pour être Verbe, n'exclut pas pour autant le Sujet: Hegel ne dit pas que seule l'Idée herméneutique d'une communication réussie au sein d'une communauté d'intersubjectivité peut apporter, dans un contexte de finitude, la solution théorique (et pratiquement risquée) au problème du " sujet " de l'histoire : il nous est honnêtement impossible de réduire le sens et la logique que l'on peut reconstruire pour l'histoire des civilisations au sens et à la

7. J. Habermas, Theorie des kommunikativen Handelns, 2 vols, Frankfurt am Main, 1981, 3e éd. 1985 (à paraître, trad. fr. par Jean-Marc FERRY, Jean-Louis ScHLegel, Théorie de l'activité communicationnelle. Paris, Fayard, 1987). 
logique qu'attribuent les acteurs sociaux ou politiques à leurs propres actions. Ce " fait de la raison historique " se transforme en fait de la domination - et plus encore, en masochisme de cette domination -, lorsqu'il est exalté dans la reddition aux "visages de l'Être" par les adversaires déclarés de la subjectivité et de l'humanisme. Mais cette apologie de l'aliénation radicale, où la "liberté de l'Être " au sens de Heidegger, liberté " époquale " de l'histoire, doit justement mesurer notre impuissance à être les sujets de cette histoire, - cette discipline apparemment salutaire de l'humiliation de la liberté humaine ne rend pas compte du fait que le Moderne ne peut pas faire autrement que de comprendre l'histoire comme le résultat de l'activité des sujets conscients ou non. C'est pourquoi l'Idée communicationnelle d'une intercompréhension entre les sujets d'une communauté d'interaction - là où se joue précisément la raison pratique - peut surmonter sur le plan théorique le paradoxe hérité de Vico : les hommes sont bien les auteurs de l'Histoire, puisque c'est bien eux qui la font ; mais ils n'en sont pas les sujets véritables, car cette histoire qu'ils font eux-mêmes, cependant, leur échappe. Dès lors que l'on prend conscience de ce paradoxe, on doit sans doute reconnaitre la nécessité méthodique d'envisager la liaison entre les monades : il n'y a pas un Sujet grand format de l'histoire, mais des individus dont l'ensemble des actions tisse la trame de l'histoire. On passe ainsi d'une métaphysique du Sujet (exemplairement représentée par Hobbes) à une métaphysique du Verbe : sauf à penser le logos lui-même comme praxis communicationnelle de sujets capables de parler, d'agir, et de se rapporter aux résultats de cet agir de façon réflexive, on postule l'autonomie d'une " raison dans l'histoire", laquelle résume certes les activités des sujets, mais transcende aussi leur propre liberté en tant qu'individus. Hegel ne conçut pas la "raison dans l'histoire" comme la performance principielle d'une "raison communicationnelle ", et c'est pourquoi il dut lui aussi - après A. Smith, Leibniz, Kant lui-même - faire fond sur le vieux topos des métaphysiques du Verbe, auquel il donna le nom de "ruse de la raison ". Ceux qui ont pu par la suite, avec l'enthousiasme de la vérité, condamner cette structure dialectique, avaient-ils une réelle solution de rechange? Plus que tout autre philosophe, Hegel a su exemplifier la formule de la Liberté de l'Esprit dans le mouvement de l'autoréflexion. Toutefois, au lieu de penser ce mouvement explicitement comme le résultat d'un processus intersubjectif, il le pensa comme un processus objectif. Bien que le mouvement de l'autoréflexion relève lui-même d'une logique de la relation je/je, il consolida ce nouvel objectivisme en recourant à l'hypostase d'une subjectivité substantielle : l'activité déterminante du Concept qui se 
réalise lui-même comme Histoire. Ainsi, la Liberté humaine - que Hegel ne cessa cependant d'affïmer - ne pouvait être clairement conciliée avec sa logique de l'Esprit. Mais la Liberté de l'Esprit fut justement pensée comme autoréflexion.

L'autoréflexion permet à l'Esprit de faire retour sur sa propre genèse formative, et de se connaître ainsi comme résultat. Du point de vue de la Logique, il s'agit de l'activité du Concept. Mais du point de vue d'une Realphilosophie de l'Esprit, il peut s'agir d'activités matérielles sensibles : dans la systématique d'Iéna, le travail, l'interaction, la représentation. Le jeune Hegel avait, en effet, mis au fondement de la réalisation de l'Esprit une systématique particulière pour caractériser les spécifications différentes de la conscience de soi au niveau du "nous", comme conscience technique, conscience pratique et conscience théorique. Les trois " puissances", instrumentation, interaction, représentation, déterminent les axes d'expérience sur lesquels se forme la conscience considérée comme le milieu dans lequel l'esprit se réalise en tant que concept, c'est-à-dire advient à lui-même sur un mode de plus en plus réflexif, tout en se chargeant de déterminations concrètes. Par-là, le " texte » que constitue l'esprit effectif maintient dans sa signification une référence latente aux trois activités fondamentales. D'un point de vue logique, celles-ci retournent au fond à trois relations que l'on pourrait encore dire "transcendantales ", dans la mesure où l'on ne peut en concevoir de plus hautes : il s'agit des trois relations à un " $i l$ » en général, à un " $t u$ » en général, et à un " je " en général - matrice grammaticale pure où s'énonce dans la différenciation des représentations contemporaines tout rapport pertinent au monde. Ces cadres transcendantaux ultimes sont toujours, bien que de façon seulement implicite, mis au fondement tant des théories de la connaissance que des théories de la société et des théories de l'activité, qui se veulent systématiques.

\section{II}

Habermas s'est intéressé à la systématique d'Iéna ${ }^{8}$. Le problème qu'il posait notamment est celui de l'articulation possible des trois dialectiques du travail, de l'interaction et de la représentation. Il put en particulier reprocher aux philosophes contemporains, qui s'inspirèrent de cette systématique, de l'avoir toujours utilisée dans un sens

8. J. Habermas, op. cit. supra n. 4. Ce texte de Habermas sur le jeune Hegel à léna date de 1967. Il semble que la «systématique d'Iéna " ait inspiré les travaux de Habermas dans les domaines de la théorie de la société et de la théorie de la connaissance jusqu'au début des années soixante-dix. 
unilatéral pour penser la réalité sociale et historique : soit comme seule logique du travail (G. Lukács) ${ }^{9}$, soit comme seule logique de l'interaction ( $\mathrm{T}$. Litt) ${ }^{10}$, soit comme seule logique de la représentation (E. Cassirer) ${ }^{11}$. Mais de façon significative, Habermas a lui-même intitulé son essai : "Travail et interaction "... Qu'était-il advenu d'une logique autonome de la représentation? Hannah Arendt ${ }^{12}$ a, quant à elle, mis en place, dans La Condition de l'homme moderne, une systématique trichotomique où les trois instances, "action", " ouvre", "travail ", se présentent comme des domaines de l'homme qui doivent être radicalement séparés, afin notamment que l' " espace de l'apparence ", espace public de l'Action où s'exprime la pureté du Politique, soit préservé des atteintes de la sphère privée où règne le Travail comme figure de la nécessité. Quant à l' Euvre, dont la conceptualité couvre normalement les catégories de la représentation ("mémoire", " tradition ", " autorité ", " religion »), elle acquiert un statut ambigu, intermédiaire, où le symbolisme de la représentation se dilue parfois dans une logique de la "fabrication ", finalement assez proche du Travail... Contre le marxisme, Habermas et Arendt s'accordent dans une certaine mesure pour affirmer l'autonomie respective du travail et de l'interaction: Habermas en affirme l'autonomie logique, tandis que Arendt soutient la position plus faible d'une autonomie réelle, bien que bafouée par l'État social et surtout par l'État totalitaire. Mais ni l'un ni l'autre n'ont explicitement articulé les trois dimensions en partant de leur autonomie logique respective. Or, c'est de là qu'il faudrait à mon avis partir : concevoir d'abord comme autonomes les trois logiques de l'instrumentation, de l'interaction, de la représentation, afin de comprendre ensuite leur articulation dynamique. Pour cela, je reconstruis assez librement les trois concepts introduits par Hegel.

9. György LUKÁcs, Histoire et conscience de classes, Paris, Minuit, 1960 ; ID., Der junge Hegel, Zurich/Wien, 1948.

10. T. LITT, op. cit. supra n. 5.

11. Ernst CASSIRER, La Philosophie des formes symboliques. 1. Le Langage; 2. La Pensée mythique; 3. La Phénoménologie de la connaissance-(Berlin, 1923, 1925, 1929), Paris, Minuit, 1972 ; ID., Essai sur l'homme (Londres, 1944), Paris, Minuit, 1975.

12. Hannah AREndT, La Condition de l'homme moderne (Chicago, Londres, 1958), Paris, Calmann-Lévy, 1961. Une réédition récente est présentée par Paul Rıcoeur, Paris, Seuil, 1985. La systématique : Action/Euvre/Travail, développée dans The Human Condition (le titre original ne parle pas d'homme «moderne ») n'est cependant qu'une partie du projet architectonique d'ensemble de Hannah Arendt. La systématique différente : Thinking/Willing/Judging a été esquissée dans The Life of the Mind, New York, 1978, trad. partielle, La Vie de l'esprit. Vol. 1 : La Pensée; vol. 2 : Le Vouloir, Paris, P.U.F., 1981 et 1983. Pour une étude d'ensemble de l'ceuvre de Hannah Arendt, André Enegren, La Pensée politique de Hannah Arendi, Paris, P.U.F., 1984. 
Le travail ou instrumentation n'est au départ qu'une catégorie abstraite de la relation de l'homme à la nature. Dans le travail, l'homme se pose lui-même comme le sujet d'une activité qui prend la nature pour objet au sens littéral d'une matière réifiée comme un neutre, un " il " (es) qui n'est lui-même aucune subjectivité, aucun « tu ». Cette relation à la nature n'est pas, en effet, la seule qui soit a priori possible; et le jeune Hegel était bien placé pour le reconnaître, du fait que ses études de théologie protestante à Tübingen furent fortement, bien qu'indirectement, influencées par la mystique juive où le thème de la Résurrection de la Nature déchue est tout à fait prégnant. Hegel "savait " que l'on peut aussi supposer une subjectivité de la nature, et s'y rapporter en conséquence d'une façon qui ne serait pas instrumentale, mais communicationnelle - autrement dit, dans une relation «je/tu ». De fait, le rapport à la nature peut être celui qu'instaure le poète qui, à l'instar du mystique, révèle autre chose que la logique d'un " il " techniquement manipulable. C'est pourquoi, si la nature est bien ce qu'elle se montre dans la technique et dans la science physique, cette réalité est toutefois celle que révèle la relation instrumentale, c'est-à-dire le cadre transcendantal d'une relation je/il. On peut par-là comprendre qu'en tant que système du mécanisme causal, la " nature " est fondamentalement l'objectivation de l'activité instrumentale ou travail. C'est ce qui permet notamment de révéler l'hypostase assumée par le concept kantien de Nature conçue comme domaine du mécanisme. Ce concept moderne de nature n'apparaît, en effet, que pour une "conscience technique " qui elle-même se formerait dans la "dialectique du travail ". Dans l'activité instrumentale se construit l'intelligence technique, qui est une connaissance des choses dans la perspective de leur manipulabilité. Hegel nommait "conscience rusée " cette conscience technique, car, dans le travail, l'homme observe la façon dont se comporte la nature, pour en tirer des conclusions " contre" la nature. Cette façon d' " observer " est à vrai dire aussi une façon de "questionner ", c'est-à-dire d'impliquer une certaine relation $« \mathrm{je} / \mathrm{tu} »$.

Cependant, ce " questionnement " de la nature ne va pas jusqu'à en interroger la finalité où pourrait se révéler un « sens". Dans la science moderne, seul le point de vue téléologique sur les êtres naturels organisés ainsi que sur le système écologique des relations finales entre ces êtres (par exemple, l'idée d'une " chaîne trophique " unissant les organismes de la biosphère) est une façon de retrouver quelque chose comme une "subjectivité " de la nature, un " ordre " qui fait sens et force le respect. Dans le monde grec antique, la téléologie était la première évidence à la fois scientifique et morale. Mais 
c'est précisément aussi dans ce rapport dit "grec " à la nature que l'homme n'était pas sujet d'une relation instrumentale à un objet réifié.

L'instrumentation permet ainsi de découvrir la réalité sous l'aspect de choses observables et techniquement manipulables. On peut, certes, tenter d'appliquer un tel point de vue au domaine - différent - des actions et des relations entre des personnes, bien que ce domaine relève catégorialement du paradigme de la Liberté et non plus de celui de la Nature. Mais cette intelligence technique ne permet absolument pas de comprendre des situations sociales dans la perspective pratique d'un participant qui doit s'entendre avec d'autres participants, afin de définir des situations communes et des plans d'action coordonnés. Lorsque, déjà, il s'agit d'organiser la division sociale du travail et d'en répartir le produit, ce n'est plus une logique du travail qui est proprement à l'œuvre, mais une logique de l'interaction.

L'interaction est la catégorie de la relation des hommes entre eux. En fonction de Hegel, on peut parler d'une « dialectique de l'interaction ", ou de la " lutte ", ou de la " reconnaissance réciproque ", ou de la "relation morale". On pourrait ainsi décrire les différents niveaux éthiques de l'interaction, depuis la lutte ouverte qui s'exprime par exemple dans la guerre ou la fatalité du crime, jusqu'à la relation morale entièrement réconciliée entre deux êtres, selon le modèle de l'amour, en passant par les activités sociales, à la fois stratégiques et discursives, qui se déroulent sous les formes de palabres, de délibérations, de négociations, de discussions menées à des fins pratiques en général et débouchant sur des accords normatifs. Cette différence entre le travail et l'interaction trouve d'ailleurs une approximation dans la distinction aristotélicienne entre technê et praxis, du fait que ces couples catégoriaux opposent une relation instrumentale " je/il " et une relation interactionnelle « je/tu ». Dans l'interaction, ce n'est pas un "objet " qui est posé pour un sujet agissant, mais un autre " sujet " avec qui l'on peut aussi communiquer. C'est, en effet, dans l'interaction que se déterminent les rôles sociaux, c'est-à-dire l'identité relationnelle, personnelle, de chaque sujet qui se constitue comme un moi individué face à d'autres moi individués. C'est dans cette logique de la reconnaissance réciproque que se fixent les statuts, se répartissent les accès différentiels à la propriété et au pouvoir, et que s'établissent par-là les normes morales externes ainsi que les institutions sociales et politiques en tant que normes et institutions légitimes. Hegel nommait ainsi "conscience reconnue" la conscience pratique dont les prédicats sont acquis sur la base d'une expérience de l'interaction médiati- 
sée par les normes. Les catégories de la conscience pratique permettent :

- premièrement, de supputer les réactions possibles d'un partenaire rationnel dans les stratégies, au regard de ses motivations, de son aptitude à les dominer, à les mobiliser ou à les transformer, ainsi qu'à tenir compte ẹt à tirer parti des motivations de l'adversaire et de ses aptitudes à les gérer - ce qui suppose un savoir psychologique ;

- deuxièmement, d'évaluer les intentions et les volitions de soimême et d'autrui, au regard des finalités légitimes qui peuvent être intersubjectivement admises dans le cadre des normes instituées, ou encore dans un autre cadre qui pourrait aussi être institué sous les conditions d'une légitimation accessible aux croyances morales en vigueur - ce qui suppose un savoir politique ;

- troisièmement, d'anticiper ce qui résulterait pour soi-même, autrui, ou la communauté tout entière, d'une transgression de ces normes, soit par ignorance ou imprudence, soit par volonté délibérée, soumettant ainsi le "criminel » à une "causalité du destin » - ce qui suppose un savoir historique.

Dans tous les cas, la relation transcendantale « je/tu " donne accès à la compréhension des fịnalités, c'est-à-dire à la " compréhension du sens ", au "Sinnverstehen " des sciences sociales et historiques inspirées par la tradition herméneutique allemande. À la différence, en effet, de la relation « je/il ", qui donne seulement accès aux " faits" correspondant à la logique des événements matériels et aux relations entre les choses, la relation " je/tu » donne, quant à elle, spécifiquement accès au "sens " correspondant à la logique des actions et des expressions ainsi qu'aux relations entre les personnes, et elle fonde la possibilité d'un savoir qui n'est pas de type analytique, mais de type herméneutique.

Cependant, l'idée qu'un "savoir", une "intelligence", une " conscience", puisse se former (différentiellement) dans le rapport instrumental au " il ", d'une part, dans le rapport interactionnel au " tu ", d'autre part, laisse encore impensé le fait qu'il faudrait déjà pouvoir présupposer un " je " - qui plus est, une instance dotée de la faculté de juger, ce qui suppose une relation spécifique du moi à luimême : « je/je ". Celle-ci n'est donnée ni dans l'instrumentation, ni dans l'interaction, mais dans la représentation. Chez le jeune Hegel, la logique de la représentation se déployait sur l'axe théorique du procès de formation de la conscience, depuis l'intuition sensible médiatisée par la conscience d'une réalité extérieure en général, jusqu'à l'autoréflexion, en passant par les niveaux de réflexivité graduels de l'imagination et du souvenir, le signe symbolique et le langage qui dénomme, 
l'entendement qui analyse et le jugement qui subsume l'image sous un concept. C'est pourquoi j'entends ici par "représentation ", non pas la simple objectivation d'images mentales, mais la faculté du symbole comme "organon de la conscience de soi ", ainsi que disait Cassirer. La représentation, puissance réflexive structurée par des symboles, ne saurait être remplacée dans cette fonction par les deux autres puissances pragmatiques de l'expérience. Pas plus que l'instrumentation, l'interaction ne permet de rendre compte de la logique propre dans laquelle se forment les représentations symboliques où les expériences déjà mentionnées - et les déterminations catégoriales qui y sont objectivement engagées, comme règles techniques et comme normes éthico-juridiques - peuvent être rendues à la conscience pour être élaborées théoriquement comme telles, et donner lieu à une tradition culturelle sans laquelle une civilisation ne serait même pas pensable. Cette compétence appartient à la troisième puissance : la représentation.

La représentation est la dimension logique qui, peut-être, mériterait le plus notre attention, car c'est elle qui porte à la conscience théorique les déterminations catégoriales de la compétence technique et de la compétence morale-pratique. C'est elle qui permet ainsi que ces catégories techniques et morales deviennent des concepts, c'est-à-dire appartiennent à une conscience en général. Les règles techniques (première "dialectique ") et les normes éthiques (deuxième "dialectique ") appartiennent certes aux déterminations d'une conscience de soi individuée, socialement constituée. Mais elles ne sont à vrai dire quelque chose pour la conscience que si elles peuvent être saisies mentalement, et réfléchies - c'est-à-dire conçues théoriquement - dans les formes symboliques qui dépendent quant à elles directement de l'activité représentative. Il convient alors d'admettre, outre la relation instrumentale-objective je/il (liée au travail), outre la relation moraleintersubjective $\mathrm{je} / \mathrm{tu}$ (liée à l'interaction), la relation mentale-autoréflexive je/je (liée à la représentation). Cette relation fonde la possibilité d'une réflexivité du sujet - et par-là, le concept de "sujet " luimême. Ce point permet de comprendre l'importance systématique de la troisième "puissance" catégorialement reliée au paradigme de l'Esprit. En effet, la représentation, relation « je/je ", est le lieu où s'élaborent théoriquement les expériences matérielles associées au travail et à l'interaction, et où se réfléchissent les objectivations de la représentation elle-même. C'est, par conséquent, dans la représentation que se forment les catégories logiques (au sens large) qui permettent d'accroître la puissance des activités instrumentales (techniques), 
morales (éthiques) et mentales (symboliques), sur la base de l'expérience totale. Ainsi, les règles techniques sont impliquées dans le travail, mais elles sont formulées dans la représentation, et deviennent par-là accessibles à une conscience de soi. Il en va de même des normes éthiques : celles-ci sont certes engagées dans l'interaction; elles structurent cette interaction et sa sphère morale, comme les règles techniques structurent de leur côté le travail et sa sphère instrumentale. Mais elles ne peuvent être rendues conscientes, c'est-à-dire thématisées, que dans les conditions de la représentation. En tant que réflexion, la représentation opère également une élaboration de ses propres objectivations : les formes symboliques; et c'est seulement ainsi que l'on obtient des concepts logiques de plus en plus purs et réflexifs à l'égard d'euxmêmes. Or, cette vue des choses porte des conséquences tant au niveau d'une théorie pragmatique de la connaissance qu'au niveau d'une théorie de l'évolution sociale. Ceux qui ont tenté, dans le cadre d'une théorie matérialiste de la connaissance, de dériver de la praxis les catégories de l'entendement, en les originant, par exemple, dans le travail social ou dans l'échange marchand (Sohn-Rethel), pouvaient certes en appeler à une intuition des "correspondances " entre la théorie et la praxis (par exemple, l'homologie entre la causalité et l'instrumentation, l'identité et l'échange d'équivalents marchands). Mais en refusant l' "idéalisme" d'une "philosophie de la conscience ", ils faisaient l'impasse sur la représentation qui est pourtant la médiation obligée pour faire comprendre comment les structures pragmatiques du travail et de l'interaction peuvent accéder au statut de structures logico-cognitives, en tant que catégories conceptuelles. De là, le matérialisme épistémologique tombe dans l'aporie d'une explication causaliste pour une genèse historique des catégories, alors qu'une telle explication ne peut être donnée qu'à l'aide de ces catégories, qui sont historiques, pour affirmer une relation qui se veut transhistorique. Or, pour espérer surmonter l'objection de ce cercle, il faut au moins supposer que les activités, dans les objectivations desquelles se déposent les expériences fondamentales de l'espèce, peuvent être formées dans une activité spécifique capable de réfléchir sur les propres représentations qu'elle tire de l'expérience, afin de les élaborer progressivement dans le concept. Le cercle logico-historique d'une théorie de la connaissance perd alors son caractère aporétique pour être fondé dans la structure de la réflexion, si du moins on en prend au sérieux le caractère spéculatif. Dans le langage du jeune Hegel, seule une "dialectique de la représentation", en vertu de la structure réflexive qu'elle suppose, serait capable de rendre compte de l'acquisition de compétences cognitives, au sens de J. Piaget, jusqu'au 
stade d'une pensée logique formelle-opérationnelle et d'une compréhension décentrée du monde. Cependant, la théorie de la connaissance n'est pas ici seule en cause. Car il y va plus fondamentalement d'une théorie du développement, aussi bien au niveau de l'apprentissage individuel (ontogenèse) qu'au niveau de l'évolution sociale (phylogenèse).

En effet, la mise en forme de l'expérience dans la réflexivité du symbole permet d'accroître de façon significative la puissance cognitive (et l'efficacité rationnelle) des activités qui sont nécessaires à la reproduction des sociétés. Ceci permet de les développer et de les rendre, en tant que cultures évolutives, susceptibles d'une histoire. Si la logique réflexive de la représentation ne devait pas intervenir comme une puissance spécifique dans le processus de formation, non seulement les formes de sociétés humaines resteraient très embryonnaires, sous nos critères de civilisation, mais encore il n'y aurait aucune histoire pensable. C'est là sans doute une évidence. Mais pour dégager cette affirmation de la trivialité du "bien connu ", le recours au raccourci d'une métaphore pourrait permettre de mieux saisir, outre le rôle, déterminant pour l'histoire, de la logique de la représentation, le schéma de son articulation dynamique dans la systématique d'ensemble: que l'on imagine la vie simple d'un homme primitif qui, le matin, se lève pour aller à la cueillette dans la forêt, y prélève des graines de plantes, qu'il tente de domestiquer en les mettant en culture dans un espace qu'il aura dû défricher, puis labourer. C'est là une expérience dans laquelle cet homme a dû se confronter à une certaine " réalité " : celle de la nature externe dont les " lois " élémentaires s'imposent dogmatiquement à lui, au sens où c'est bien la nature qui lui « dicte " la façon de s'y prendre avec elle pour parvenir efficacement aux fins que l'homme cherche à réaliser par son travail. L'homme doit travailler, et c'est dans cette logique de l'instrumentation qu'il expérimente pratiquement, selon la logique des essais et erreurs, les lois de la nature, des lois qu'il ne saurait ni intuitionner immédiatement ni déduire $a$ priori. Vers midi, l'homme arrête le travail, car il doit négocier avec les autorités de sa famille et de son clan pour obtenir le droit d'exploiter une plus grande parcelle de terrain. La négociation s'engage; l'homme se heurte à des résistances et à des objections (des réticences étayées) contre lesquelles il argumente, puis des refus conditionnels qu'il doit assouplir en proposant des contreparties. Là encore, il fait l'expérience d'une "réalité ". Mais cette réalité n'est plus du même genre que la précédente, car elle n'est pas cette fois l'obstacle que dressent contre lui les réquisits de la nature (dont il fit une expérience 
dans le travail), mais l'obstacle différent que dressent contre lui les réquisits de la volonté de ses protagonistes. L'expérience de cette réalité, il ne peut la faire que dans l'interaction.

Cependant, il s'agit dans les deux cas d'expériences brutes qui pourraient ne laisser à cet homme aucun souvenir fixé, car elles ne laisseraient pas de traces durables dans sa représentation. On pourrait imaginer que ses activités de la journée aient été aussitôt refoulées ou simplement non retenues mentalement, de sorte que l'être auquel on impute des expériences ne pourrait en aucune façon tirer des leçons de celles-ci. La représentation permet précisément cette remémoration imaginative, qui élabore dans la conscience les résultats de l'expérience, et en fait une expérience partagée en les transmettant grâce à la communication. Ainsi se constituent les traditions de base, dont l'essence communicationnelle assure le lien des hommes entre eux et à eux-mêmes comme structure réflexive d'un savoir-de-soi nécessairement dirigé vers un antérieur. D'où surgit le passé historique, toujours actualisable, mobilisable au présent pour informer l'avenir possible. Dans les sociétés traditionnelles, ce moment de la remémoration a une importance particulière : c'est l'importance du récit, de la narration, de la tradition orale. Celle-ci doit être prise comme un enseignement grâce auquel se conserve et se transmet l'expérience acquise. Bien sûr, la matière de cette expérience est aussi la tradition elle-même, une fois qu'elle est archivée dans ce "patrimoine spirituel de la civilisation" dont parlait S. Freud. C'est encore l'importance que l'on y accorde au rêve, qui, pour les sociétés traditionnelles, est souvent le lieu privilégié de la révélation. Le récit comme le rêve expriment la fonction de la représentation pour l'apprentissage social. Suivant la métaphore ici suggérée, l'homme travaille le matin, s'engage dans l'interaction l'après-midi, et raconte (représente), le soir, ce qui s'est passé dans la journée, tandis que sous une forme ou sous une autre il pourra en rêver la nuit. Le récit du soir et le rêve de la nuit traduisent métaphoriquement la façon dont la représentation métabolisera dans le symbole le sens de l'expérience acquise au contact de la réalité - des « trois réalités" - et tirera les leçons de ces expériences de la veille pour les réinvestir, pourrait-on dire, "le lendemain", dans le travail et l'interaction - augmentant ainsi le potentiel cognitif au sens large, grâce auquel l'efficacité vis-à-vis de l'environnement naturel et social pourra être accrue.

Or, en augmentant ainsi le potentiel rationnel d'interrogation du réel, on augmente par-là même la quantité des réponses, c'est-à-dire l'intensité de l'information. Ce modèle de synergie peut être transposé à la phylogenèse : avec l'accès au premier stade logique de la forma- 
tion de l'esprit, c'est-à-dire celui qui, dans l'évolution des compréhensions du monde, doit correspondre aux Mythologies, la réalité acquiert une cohérence temporelle qui correspond sans doute à la possibilité culturelle de fixer et légitimer des pratiques dans des traditions narratives. Cette cohérence qu'exprime le récit de l'épopée et du mythe peut s'approfondir dans la représentation de totalités finalisées et hiérarchisées, jusqu'à la conscience d'un ordre régulé du monde stable, où les forces invisibles ou inconscientes auxquelles s'affrontent les hommes sont suffisamment dominées, tant par l'action que par la représentation, pour s'organiser en une hiérarchie - ce qui correspondrait au deuxième grand stade de civilisation, celui des Cosmologies. Cependant, cet ordre hiérarchisé du monde permet par suite d'autonomiser les uns par rapport aux autres des domaines pertinents de réalité et de sens, et de parvenir ainsi à une différenciation entre des ordres logiques du réel, c'est-à-dire à la notion de légalités ontiques et déontiques en même temps, à la notion de lois, sur laquelle s'organise le troisième stade, celui des Ontologies.

Dans la chronologie des cultures occidentales, l'apparition postérieure des Théologies, au sens du monothéisme judéo-chrétien, ne possède pas par elle-même la signification d'une postériorité logique (par rapport à l'ontologie d'Aristote), car son apparition historique obéirait à une autre généalogie conceptuelle, originée dans le judaïsme, et elle se situe par-là sous le signe de la contingence. Ce n'est que rétroactivement, au prix d'un travail conceptuel et argumentatif de plusieurs siècles, que le monde chrétien a pu intégrer à sa théologie l'ontologie grecque, et déterminer ainsi de nouvelles possibilités logiques pour la suite : avec l'avènement du Moderne, les Métaphysiques pré-critiques, puis les Architectoniques de la raison, aujourd'hui, les Systématiques de sciences réflexives post-philosophiques, représentées notamment par les théories de la connaissance et des sciences (Épistémologies), les théories de l'action et des activités rationnelles (Praxéologies), les théories du langage et de la signification (Sémiologies). Par des approches et des stratégies conceptuelles différentes, ces pointes contemporaines de l'autoréflexion mettent à jour les relations fondamentales qui s'organisent sous le système de référence des trois personnes pronominales. Non seulement elles réfléchissent toujours les expériences fondamentales de l'espèce, mais elles en explicitent en outre les conditions générales formelles. Bien sûr, l'esquisse proposée ici s'inscrit dans la ligne de ces tentatives. Reste encore " substantiel " l'enjeu de concevoir l'articulation de l'expérience. À cet égard, l'articulation dynamique des trois logiques d'activité est un problème à résoudre pour une théorie du développement qui serait introductive au concept 
d'histoire, bien que l'histoire soit irréductible à une logique du développement. Mais un autre problème est celui de leur articulation logique. Ce problème doit être, quant à lui, résolu pour une théorie de la société fondée sur l'idée que la société se constitue dans des systèmes d'activités organisées. Comment les trois moments du travail, de l'interaction, de la représentation, peuvent-ils ne pas être trois moments radicalement séparés?

Pour tenter de répondre à cette question, on pourrait accorder en prémisses que chacune des trois activités constitutives produit des objectivations spécifiques: les objectivations de l'instrumentation sont des productions; les objectivations de l'interaction sont des institutions; les objectivations de la représentation sont... des représentations.

a) Comment les objectivations du travail sont-elles médiatisées par celles de l'interaction et de la représentation? Elles le sont d'abord par la représentation, parce que toute production méthodique suppose une distance entre la conception et la réalisation : le projet est représenté pour être exécuté ; et c'est ce qui fait le principe de toute technologie. Elles le sont ensuite par l'interaction, dès qu'il faut parler de travail social, de produit social (et de revenu socialement réparti). K. Marx a, comme on sait, montré que toute production sociale, toute marchandise, " exprimerait " les " rapports de production " qui lui confèrent une signification sociale. La production n'est pas seulement opérée sous des conditions technologiques déterminées; elle l'est aussi sous des conditions politiques déterminées; et c'est ce qui fait le principe de toute économie.

b) Comment les objectivations de l'interaction sont-elles médiatisées par celles de l'instrumentation et de la représentation? Elles le sont déjà par la représentation : d'une part, la représentation comme telle est logiquement présupposée à la reconnaissance réciproque qui permet par suite de répartir les rôles sociaux et de définir les statuts sociaux - ce dont l'étude est classiquement l'affaire de la sociologie; d'autre part, aucune interaction sociale ne peut déboucher sur l'établissement d'institutions sans présupposer la possibilité au moins virtuelle de discussions et de négociations, c'est-à-dire une interaction médiatisée par des symboles et régulée par des prétentions à la légitimité - ce dont l'étude est classiquement l'affaire de la politique. Elles le sont ensuite par le travail et la représentation, parce que l'un des aspects fondamentaux de l'échange social n'est pas seulement l'échange langagier, mais aussi l'échange marchand, c'est-à-dire une interaction médiatisée par des productions et représentée par le « langage " fonctionnel de signes monétaires - ce par quoi l'économie poli- 
tique au sens large devrait pouvoir thématiser son propre accès au spectre total des trois dimensions logiques.

c) Comment, enfín, les objectivations de la représentation sont-elles médiatisées par celles de l'instrumentation et de l'interaction? Elles le sont - de façon plus complexe - comme résultat analytique d'une réflexion sur la structure logique incarnée dans les « objets » constitués dans le travail et l'interaction. De ces objectivations, la représentation dégage donc, soit des règles techniques et des lois technologiques ou scientifiques (pour l'instrumentation), soit des normes sociales, et des principes éthiques ou juridiques (pour l'interaction). Mais en raison de sa nature réflexive, la représentation peut aussi réfléchir sur ses propres représentations symboliques, dont elle dégage les noyaux logiques, comme catégories conceptuelles, ainsi que les fondements critiques, comme principes de la réflexion. $\mathrm{Si}$ la représentation n'était pas médiatisée par l'instrumentation, d'une part, l'interaction, d'autre part, elle n'aurait accès ni aux catégories de la technique et de la science, ni aux catégories de l'éthique et du droit. Et si elle n'était pas médiatisée par ses propres objectivations : la « matière " constituée par les formes symboliques mobilisables, elle n'aurait pas accès aux catégories de la logique et de la critique.

Jean-Marc FERRY, Institut Raymond-Aron, Université Goethe de Francfort. 